\title{
Multi-Distance Veins Projection Based on Single Axis Camera and Projector System
}

\author{
I Putu Adi Surya Gunawan', Riyanto Sigit' ${ }^{2}$, Agus Indra Gunawan ${ }^{3}$ \\ Department of Electrical Engineering1,3 \\ Department of Informatics and Computer Engineering 2 \\ Politeknik Elektronika Negeri Surabaya \\ Jalan Raya ITS, Sukolilo, 60111, Indonesia \\ Email: iputuadisurya@pasca.student.pens.ac.id,\{riyanto,agus_ig\}@pens.ac.id
}

Received March 8, 2019; Revised May 19, 2019; Accepted June 21, 2019

\begin{abstract}
Every person has different location of veins, some veins are easily detected because it is visible due to thin tissue, and the other are invisible. This different location of veins causes intravenous access procedures and the process of intravenous therapy become longer. Multi-distance veins projection aim to simplify the measurement process where the device and object do not have to be at a certain distance. Some research that has been done especially for real-time vein projection does not conduct how the characteristics of projection at different distances. In this paper, we propose a method for performing multi-distance real-time back-projection by using the intersection between camera and projector. This method equiped with an ultrasonic distance sensor to identify the projection characteristic in any distance. In its implementation, this method is able to project at a distance of 20-40 cm with a maximum projection error of $0.6 \mathrm{~mm}$. The measurement angle tolerance between the object and the device is \pm 5 degrees with a maximum error of $0.7 \mathrm{~mm}$.
\end{abstract}

Keywords: veins, back-projection, ultrasonic distance sensor.

\section{INTRODUCTION}

Every person has different location of veins, some veins are easily detected because it is visible due to thin tissue, and the other are invisible. This different location of veins causes intravenous access procedures and the process of intravenous therapy become longer.

Currently, there are medical devices known as vein contrast enhancers (VCE) to help with the process of finding veins. By using the principle of absorption of the infrared spectrum, this tool can help visualize the veins. In a study of the success rate of the first trial in finding veins, the use of VCE in 
patients increased the success rate to 56.3 percent in patients with high difficulty levels [1]. Currently there are some vein viewer products such as VeinViewer, AccuVein, and VascuLuminator which have an important role however the price is still too expensive [2].

Previous studies that discussed the visualization of veins for medical purposes mostly focused on processing vein images without back-projection. Back-projection has advantages where the results of visualization are immediately visible on the surface of the skin. Therefore, vein visualization process becomes simpler because there is no need an additional screen to observe the vein. Several studies also focused on back-projection to visualize the veins directly on the skin. However, the back-projection that performed are limited at a fixed distance. Those fixed distance limitation can cause the visualization process less flexible. An idea to develop a back-projection method that able to perform at a certain distance range is expected to increase the visualization flexibility. By knowing the projection characteristics at various distances, it will be possible if the projection results will adjust based on distance. Furthermore, the process for visualization will be simpler because it does not have to fit at a fixed distance.

This paper proposes a back-projection method and performs observation on projection characteristic in multi-distance $(20-40 \mathrm{~cm})$. With a camera and projector placed on a single axis, the intersection between the camera view and the projector view was utilized as projectable area. An ultrasonic distance sensor was used in this study to determine the characteristics of the projection location and adjust the projection results at multi-distance. Before entering the back-projection stage, the vein image acquired with a combination of NoIR camera and NIR LEDs processed through the preprocessing and segmentation stages. Some image processing algorithms such as filtering, high-boost filter, adaptive threshold etc. were performed to obtain a clear vein pattern. The application of the infrared pass filter on the NoIR camera was very useful in preventing feedback (the camera captures the image that already projected) that make a loop and distracting the original image.

\section{RELATED WORKS}

The first systematic study on vein image back-pojection was conducted by Zeman [3]. The main idea of their method was to re-project what the camera captures. The camera and projector must be at one point to make the projection result at the right location. In order to accomplish that concept, the camera and the projector were placed on a different axis and separated by a hot mirror. This projection method can be seen in Figure 1.a.

Dai et al. [4] examined how to project vein images to simplify the method proposed by Zeman. In their research, the camera and projector were placed in the same axis thus there was no need separation between camera and projector using hot mirror. This simpler back-projection method shown in Figure 1.b. They mentioned that their method produced good results with projection shift of $0.8 \mathrm{~mm}$. However, there is still considerable ambiguity with 
regard to the distance change between device and object affecting the backprojection result.

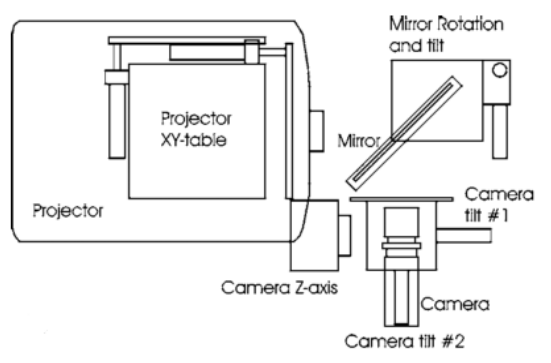

(a)

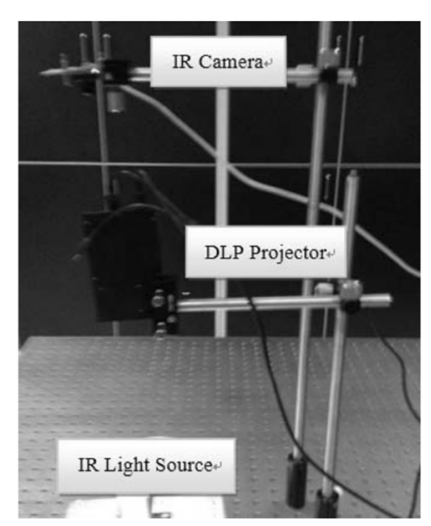

(b)

Figure 1. Previeous method on real time back-projection projection by Zeman [3] and Dai et al. [4].

Ai et al. [5] conducted a research about vein back-projection using 2 cameras and projector. The device setup shown in Figure 2, it uses a dual camera system to obtain the image of vein depth. Although the results of the reconstruction of veins could be resolved, their approach is not well suited for real-time systems because of very long computation time.

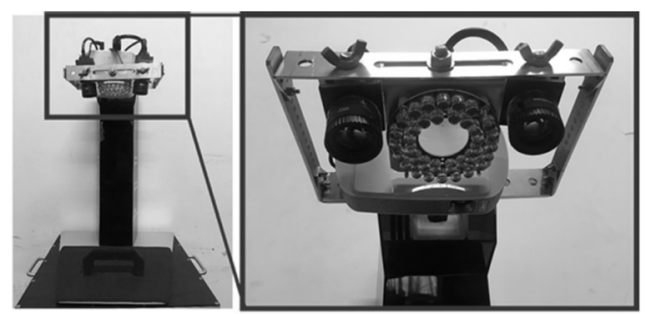

Figure 2. Back-projection method by Ai et al. [5].

Since the application of this tool focused on mobility and real-time system, previous study [6] has developed image processing algorithms on a single board computer.

\section{ORIGINALITY}

In previous studies, there were various methods for back-projection of veins image. However, none of those studies has clearly examined or discussed the effect of distance on projection results and only perform back-projection at a fixed distance.

In this article, the principle of the intersection of camera view with projector view is used to perform back-projection. Based on this principle, the position of the camera with the projector can be placed parallel in single axis as shown in 
Figure 8. Meanwhile, a distance sensor is included to find out the characteristics of the projection location at each particular point between the acquisition device and the object. By knowing these characteristics, it is expected that the system will be able to adjust the projection even though the distance between device and object changes. In addition, the $650 \mathrm{~nm}$ IR pass filter is used to block the feedback (projected image generated by the projector that assumed as visible light). Therefore, the feedback that causes the vein image overlapped and noisy does not occur.

\section{SYSTEM DESIGN}

In this study, the camera (RPi NoIR camera) and the distance sensor were used as input of the system. The input will be processed by the microprocessor (Raspberry Pi 3B) and produce output to the projector (D5S mini DLP Projector) and NIR LED as shown in Figure 3. A microcontroller was used to filter the output data of the ultrasonic distance sensor by applying a moving average filter. The filtered distance data was transmitted to the microprocessor using serial communication. As a core of the system, the microprocessor has a function to regulate input-output and run the image processing algorithms.

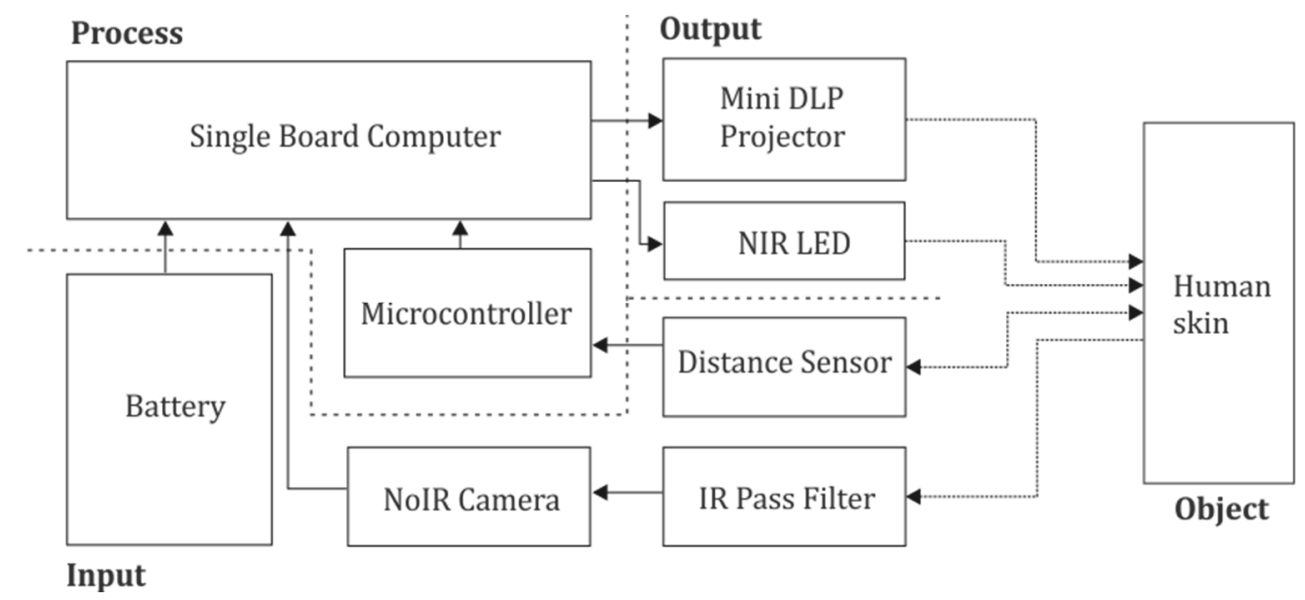

Figure 3. Hardaware design. 


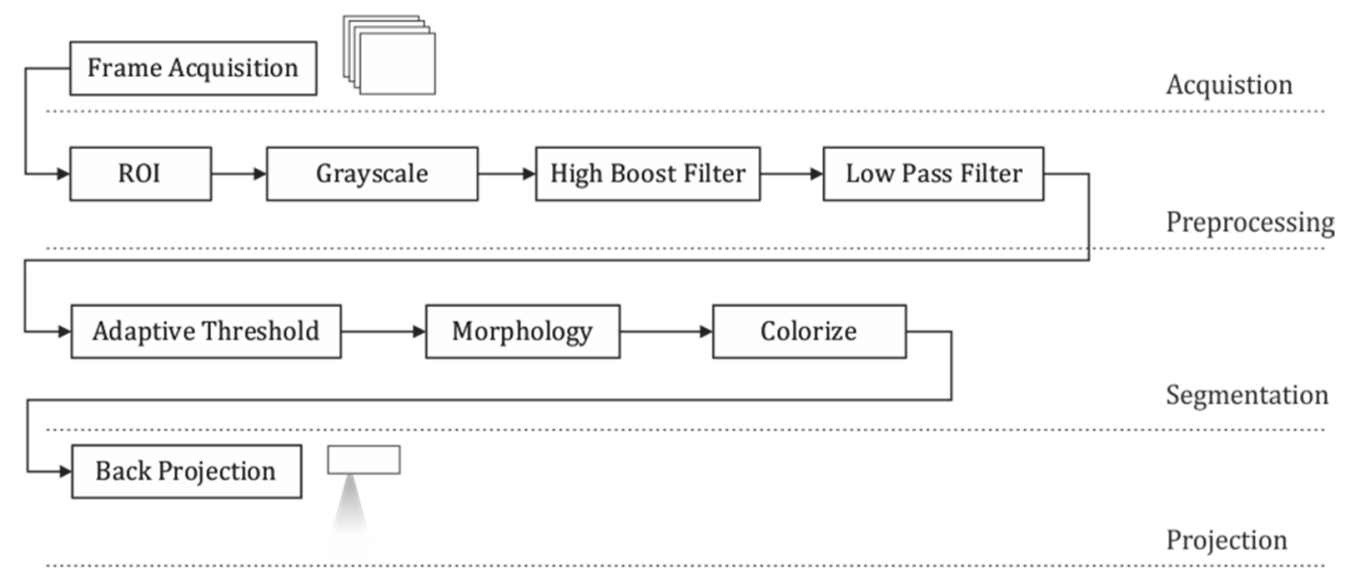

Figure 4. Software design.

Figure 4 shows the software design of this system. There are 4 stages consist of acquisition, preprocessing, segmentation and back-projection. At the stage of data acquisition, vein images were taken by a reflection method using $720 \mathrm{~nm}$ infrared light and filtered by $650 \mathrm{~nm}$ infrared pass filter. Vein images that have been obtained need to be enhanced at the preprocessing stage to facilitate the segmentation process. Vein images that already enhanced in the preprocessing stage were ready to segmented in segmentation stage and the last process was to re-project the segmented vein image based on the method that proposed in this study. All the image processing algorithm were performed using OpenCV with Python programming language.

\subsection{Vein Image Acquisition}

Study on the characteristics of light absorption in blood contents has been carried out by Prahl [7]. In his research, the range of light spectrum from $300 \mathrm{~nm}-1000 \mathrm{~nm}$ applied to oxyhemoglobin and deoxyhemoglobin. As shown in Figure 5, the difference in the absorption of light between oxyhemoglobin and deoxyhemoglobin appears at a wavelength of $600 \mathrm{~nm}-720 \mathrm{~nm}$ (near infrared rays) and the furthest difference occurred around $680 \mathrm{~nm}$. Since the veins contain deoxyhemoglobin and it has high absorbance at $680 \mathrm{~nm}$ wavelength, it was possible to capture the veins using a camera and illuminated by NIR LED. 


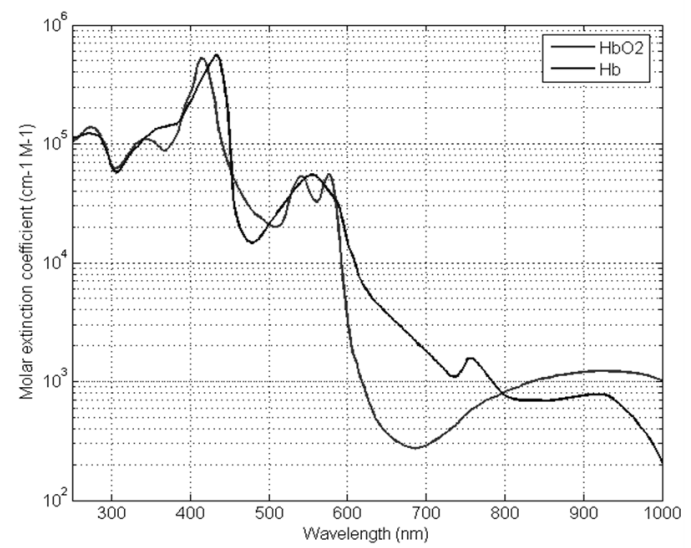

Figure 5. Difference in light absorption between oxyhemoglobin and deoxyhemoglobin [7].

There were normally 2 methods known as reflection mode and penetration mode that used to obtain a vein image as shown in Figure 6. Penetration mode has been widely studied and developed into several studies [8], [9]. The penetration method does not provide flexibility in measuring because the target and source must remain in the same position. While this paper focuses more on measurements with various distances. Therefore, the use of reflection mode is more appropriate to use in this system.
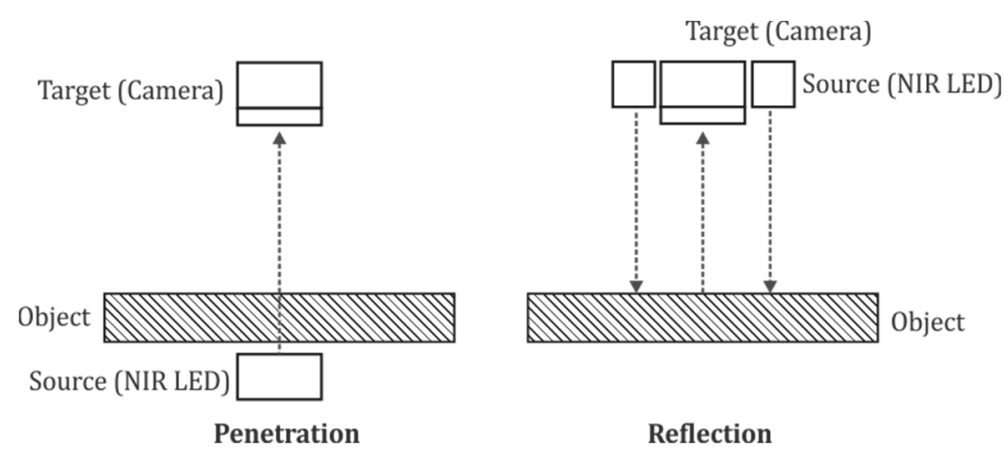

Figure 6. Comparison between penetration and reflection method to obtain vein image.

\subsection{Preprocessing}

Determine the region of interest (ROI) is the first step performed in the preprocessing stage. Based on the proposed method shown in

Figure 8, the intersection between the camera view and projector view only occurs on the bottom of camera view. Therefore, the ROI is determined at the bottom center of the frame with a size of $340 \times 180$ pixels as shown in Figure 7. 


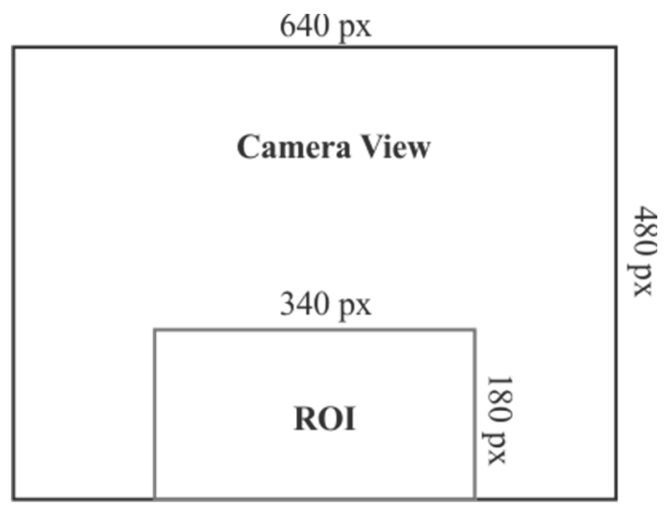

Figure 7. Region of Interest size and position.

The original image obtained by the camera has an RGB format. The grayscale conversion was performed because the information on veins images contains only dark (veins) and bright (skin). Due to the fact that the grayscale process has not produced a strong difference between the vein and the skin, the image enhancement needs to perform. Several studies, for instance [10] and [11], have been carried out the histogram equalization method to make a strong difference between veins and skin. In order to reduce lost information, another method such as contrast limited adaptive histogram equalization (CLAHE)[8] and high-boost filter were more suitable to enhance the veins image. High-boost filter giving a better result than CLAHE even its computation time was a little bit slower [6]. Therefore, a high-boost filter was applied in this paper. The working principle of the high-boost filter is to substract the image with the results of the low pass filter from the image. The use of lowpass filters could be either a median filter [12] or a Gaussian filter [13]. High-boost filter can be expressed as $G_{(m, n)}$, where $A$ is the gain and $F_{(m, n)}$ is the original image.

$$
\begin{aligned}
& G_{(m . n)}=A \cdot F_{(m, n)}-\operatorname{lowpass}\left(F_{(m, n)}\right) \\
& G_{(m, n)}=(A-1) \cdot F_{(m, n)}+\left[F_{(m, n)}-\operatorname{lowpass}\left(F_{(m, n)}\right)\right] \\
& G_{(m, n)}=(A-1) \cdot F_{(m, n)}+\operatorname{highpass}\left(F_{(m, n)}\right)
\end{aligned}
$$

Since the enhancement process amplifies the black and white, there is a possibility that the noise also amplified. Therefore, a filtering process such as a median filter or Gaussian filter was performed to minimize the noise.

\subsection{Segmentation}

Segmentation is the process of separating vein patterns from the skin. In this study, preprocessed image was segmented using the thresholding method. Since the thresholding value is global, a lot of information can be lost because 
of the process. Therefore, adaptive thresholding [14] was adopted to perform segmentation in this paper. In order to determine the threshold value in each block, the mean threshold value was used rather than Gaussian threshold value due to faster computation time. The mean threshold value $T_{(x, y)}$ expressed in Equation 4. $n_{(i, j)}$ is the sample window with size of $i \times j$ while $n_{(x, y)}$ is the original image with size of $x \times y$.

$$
T_{(x, y)}=\frac{\left(\sum_{i=0}^{I-1} \sum_{j=0}^{J-1} n_{(i, j)}\right)-n_{(x, y)}}{I J-1}
$$

A morphology operation was performed in order to reduce the noise in a binary image from the result of adaptive thresholding. Combination of morphology opening and closing was used to deal with noise. Morphology opening can be expressed as:

$$
A \circ B=(A \oplus(-B)) \oplus B
$$

Where $A$ is the result of adaptive thresholding and $B$ is the structuring element. This method was able to eliminate the noise but also makes the vein pattern disappear in some parts. Therefore, morphology closing is used to restore the vein pattern and it can be expressed as:

$$
A \bullet B=(A \oplus B) \oplus(-B)
$$

Colorization is the process of coloring the segmentation result before it projected. The black color cannot be projected by a projector. While the white color was blended with the object color when projected. Therefore, a colorization process was performed to improve the clearness of visualization.

\subsection{Back-projection}

Back-projection is a process to display the vein patterns that has been segmented back to the surface of the skin. As mentioned in Section 3, we proposed a method for projection without using hot mirrors. The proposed method has components such as a projector, distance sensor, and a NoIR camera. These components are placed in such a way to make an intersection between the camera and projector as shown in figure 8 . 

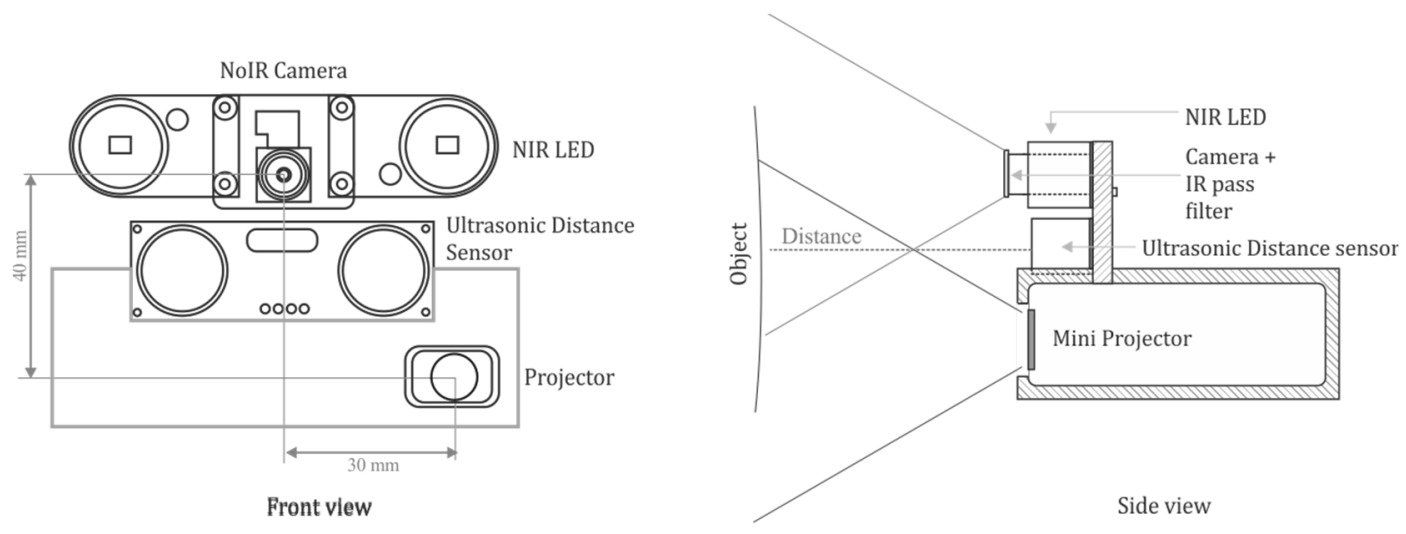

Figure 8. Proposed back-projection method

Performing back-projection calibration directly to the skin is very difficult to do because it requires an object that remains in position and rotation. Therefore, a simulation object was created to observe the characteristics of back-projection. Based on a research conducted by Tserevalakis et al. [15] in

Figure 9, it shows that blue (ultramarine blue) has a high absorbance level when given a wavelength of around $700 \mathrm{~nm}$. This color is in accordance with the characteristics of veins that contain DeoxyHb because it absorbs NIR light based on the molar absorption coefficient in Figure 5. Meanwhile, chrome yellow has a low absorbance level when given NIR light and it is in accordance with $0 x y H b$ from Figure 5. This color tends to reflect back the NIR light thus it will not be visible to the camera.

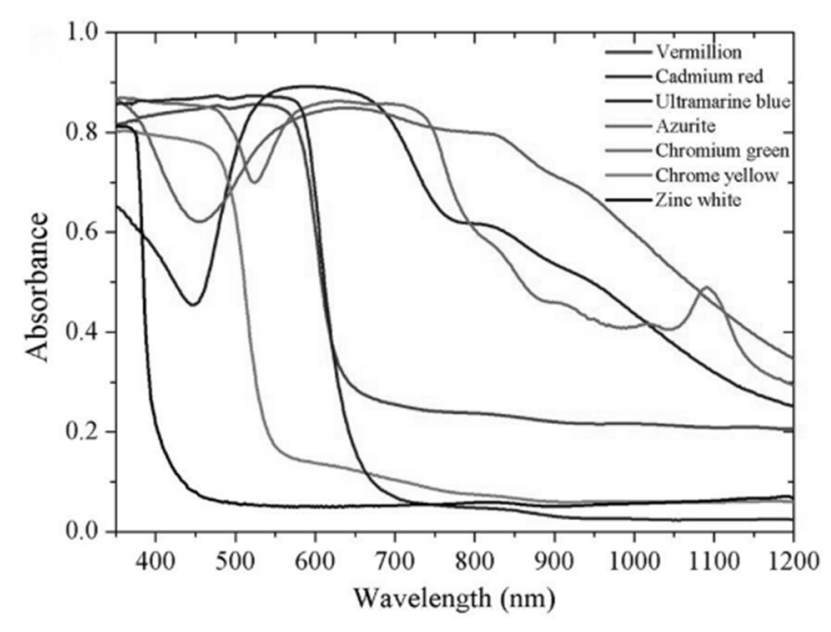

Figure 9. Photoacoustic imaging results from several sample paint [15].

A test was conducted in order to prove the result of Figure 9. In this test, some colors (blue, green, red, yellow) are printed in HVS paper as shown in

Figure 10.a and observed using veins acquisition method. 


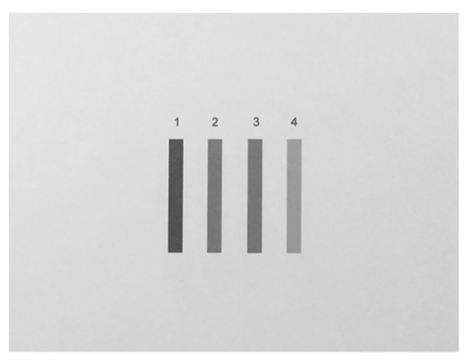

(a)

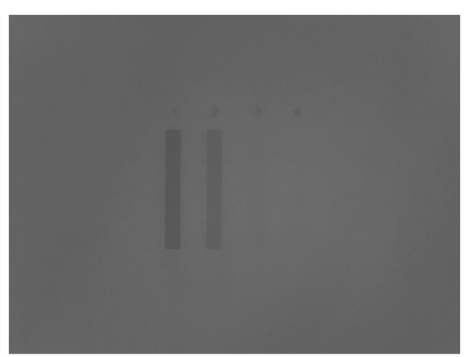

(b)

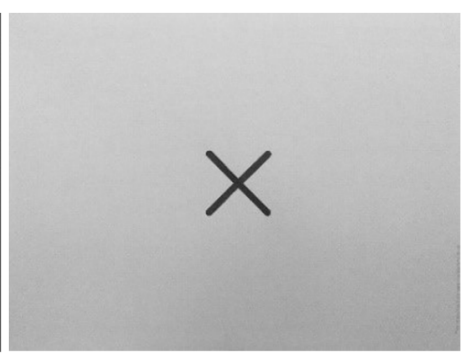

(c)

Figure 10. (a) Some color samples that used to simulate veins; (b) Result of captured image using NoIR camera and illuminated by NIR LED; (c) The object used as a simulation uses the most dominant absorbed color.

Figure 10.b shows the color which has high absorbance will visible on the camera view while the color with low absorbance is not visible. Therefore, a simulation object was created by using HVS paper and using blue color as simulated veins as shown in

Figure 10.c. This chrome yellow color was used as a grid on the object to simplify the measurement process if there is a shift in projection. Size of the line was set to $4 \mathrm{~mm}$ because the veins in the arms have sizes in a range of 0.7 - $7.3 \mathrm{~mm}[16]$.
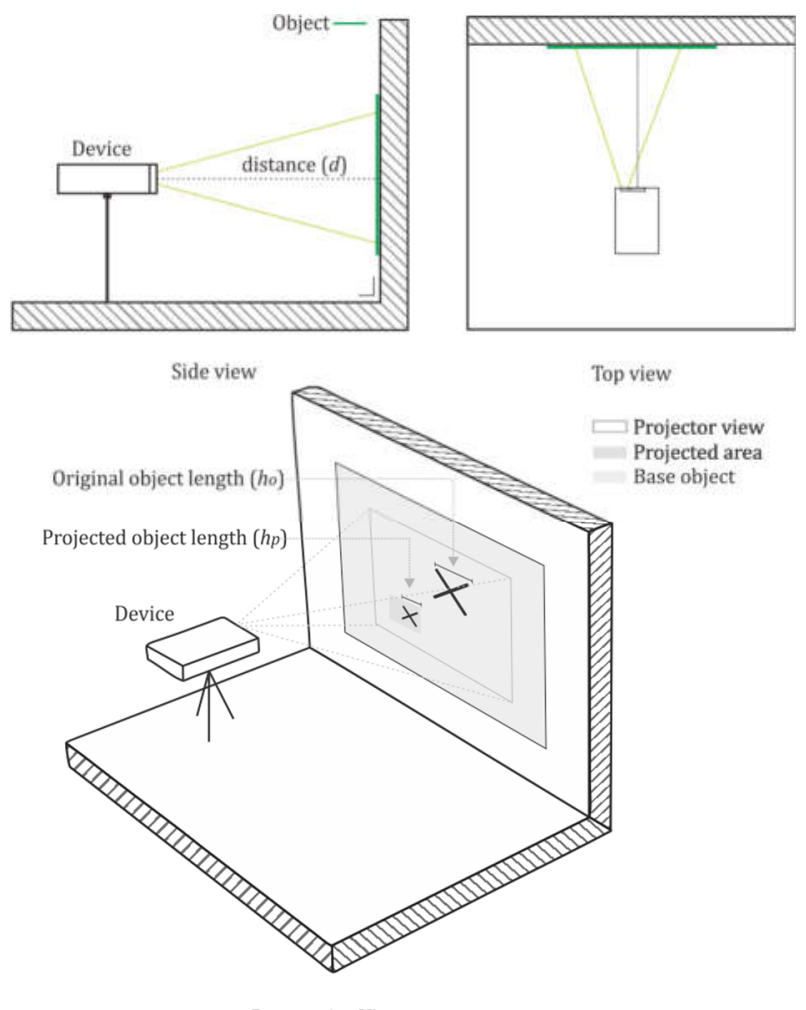

Perspective View

Figure 11. System design for testing back-projection. 
Back-projection method is performed based on the system design that is shown in

Figure 11. While the implementation is shown in Figure 12.a and Figure 12.b. The results obtained were not good where the projected image was captured again by the camera (feedback) and produce a loop as shown in Figure 12.c. The image becames distracted due to interference by the projection result. A $35 \mathrm{~mm}$ film that has passed the exposed and developed process was used by Juric [2] to solve the feedback problem. Whereas in this paper, $650 \mathrm{~nm}$ DSLR IR pass filter was used to block visible light and pass infrared light. By applying this filter, the captured image would not be interfered by the projection result.

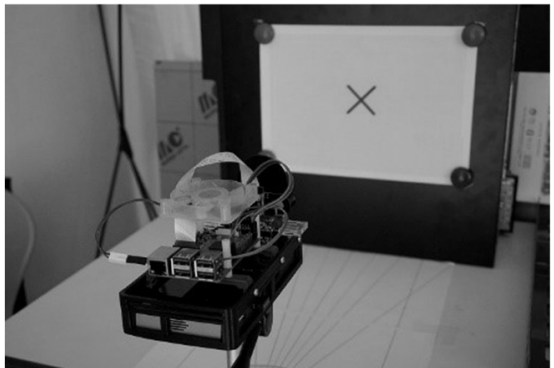

(a)

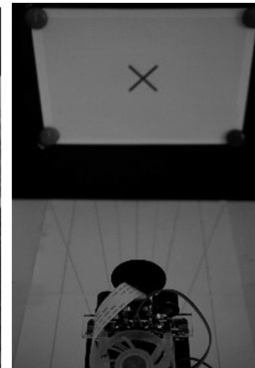

(b)

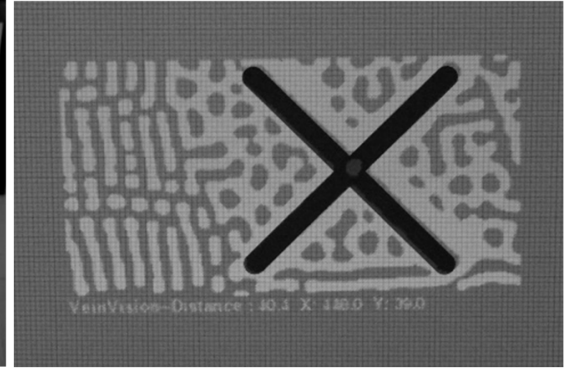

(c)

Figure 12. Back-projection test setup. (a) Side view; (b) Top View; (c) Feedback on the projection result.

\section{EXPERIMENT AND ANALYSIS}

\subsection{Result of vein image processing}

The results obtained by the camera are shown in Figure 13. The difference between those two images are the use of an IR pass filter (left) and without an IR pass filter (right).

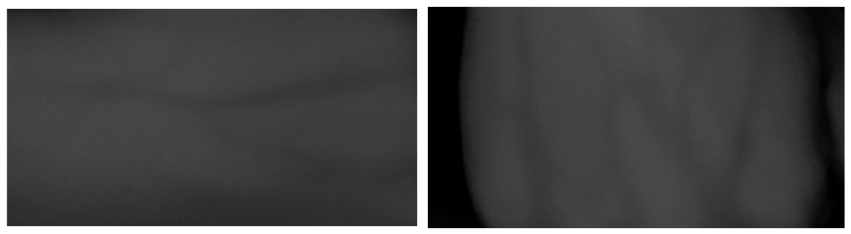

Figure 13. Frame captured by NoIR camera.
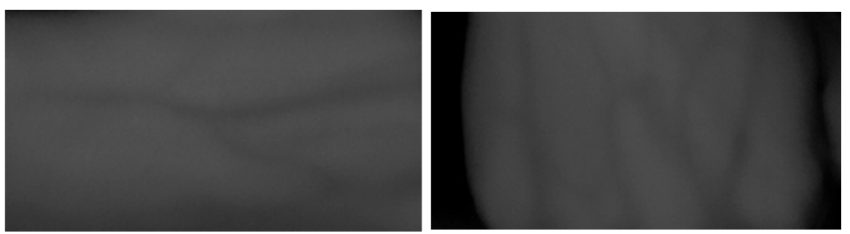

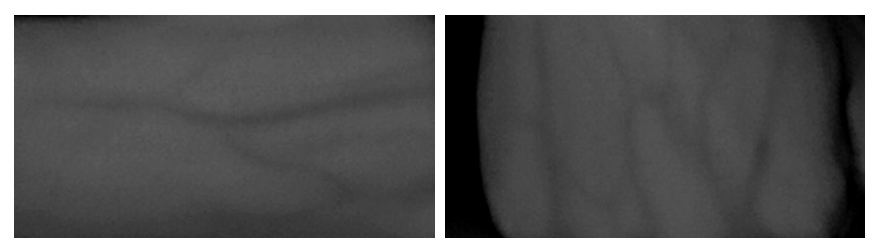

Figure 14. Preprocessing result. (top) Grayscale; (bottom) high-boost filter.

Figure 14 shows the grayscale results. There was no difference between using an IR pass filter or not in grayscale results. However, the use of the IR pass filter here was intended to block the light reflected by the projector. Meanwhile, the high-boost filter was able to increase the difference between veins pattern and skin.

Since the results of preprocessing already has a clear difference between the vein pattern and the skin, it will be easier to obtain a good segmentation result. The mean threshold was able to minimize pattern loss in the segmentation process.

Figure 15 shows the segmentation results using the mean threshold value and the Gaussian threshold value.
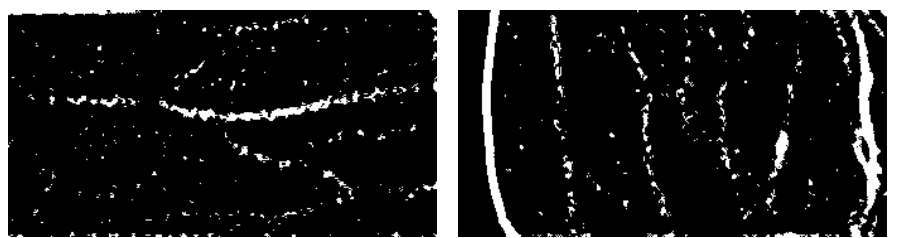

(a)
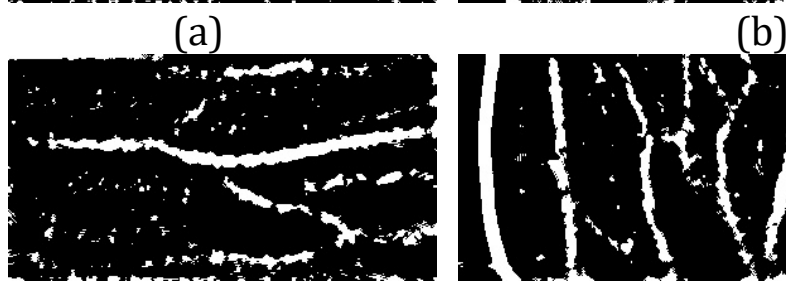

(c)

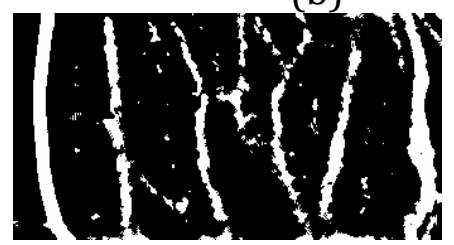

(d)

Figure 15. Segmentation result; (a, b) mean threshold; (c, d) gaussian threshold.

From the results of the segmentation process, it can be seen that the mean threshold value provides more information on vein patterns. In other hands, the usage of Gaussian threshold value was less suitable because of less information and slower computation time. 


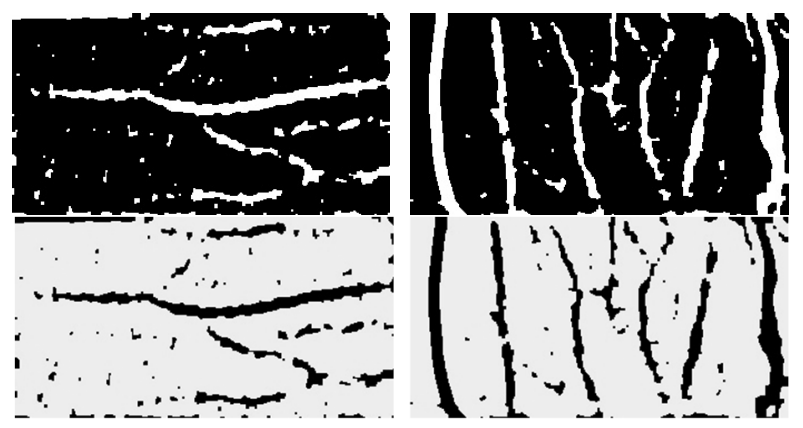

Figure 16. Segmentation result; (top) Morphology operation result on removing noise after segmentation process; (bottom) colorization result to improve clearness of visualization.

Figure 16 shows the morphology operation results (top) in eliminating the segmented noise. These methods cannot be maximized because it will make the pattern disappear. In previous research [6], the use of contour areas to eliminate noise was very strong. However, because the execution time was long enough, it is not suitable for real-time systems. Segmentation results are colored as shown in

Figure 16 (bottom) before entering the back-projection stage.

Table 1. Execution time for each image processing algorithm

\begin{tabular}{|l|l|l|}
\hline Stage & Methods & Time (s) \\
\hline Acquisition & Frame acquisition & $8.48^{*} 10^{-6}$ \\
\hline \multirow{3}{*}{ Preprocessing } & Region of Interest & 0.000421 \\
\cline { 2 - 3 } & Grayscale & 0.001765 \\
\cline { 2 - 3 } & High-boost filter & 0.022105 \\
\hline \multirow{3}{*}{ Segmentation } & Low-pass filter & 0.006425 \\
\cline { 2 - 3 } & Adaptive thresholding & 0.005021 \\
\cline { 2 - 3 } & Morphology operation & 0.015122 \\
\cline { 2 - 3 } & Colorization & 0.003191 \\
\hline Total time & Determine projection location & 0.005532 \\
\cline { 2 - 3 } & Put image on specific location & 0.006936 \\
\hline
\end{tabular}

Table 1 shows the execution times for each image processing algorithm to visualize the vein. Each method of image processing already chosen that have better result and faster computation time. This combination of image processing algorithms took about 0.0665 seconds to process 1 frame. Since this system visualizes the veins for intravenous therapy, $15 \mathrm{fps}$ is fast enough because the object will remain static during visualization.

\subsection{The result of back-projection}

The processed frame size was $340 \times 180$-pixel. When projected, the frame size experienced enlargement and reduction based on projection distance. An 
observation was carried out by performing projections with a distance of 20$50 \mathrm{~cm}$ to find these characteristics.

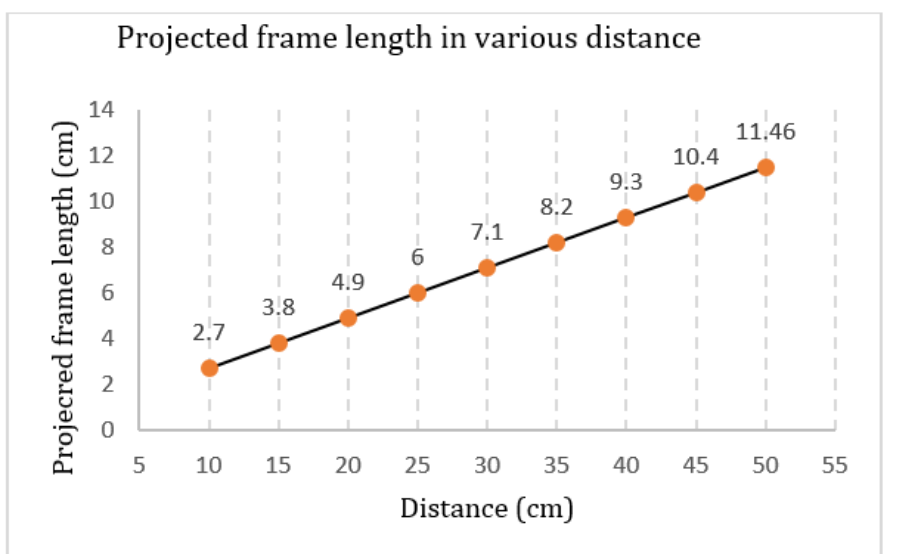

Figure 17. Projected frame length characteristic in various distance.

From

Figure 17, it shows that the smaller the distance, the smaller the projection frame length. Since the size of the projection frame was affected by distance, it is necessary to measure whether the size of the object also has such characteristics. The object to be measured has several lengths ranging from 5 to $2 \mathrm{~cm}$ with a step of $0.5 \mathrm{~cm}$. This observation was performed at a distance of 40-10 $\mathrm{cm}$ with a $5 \mathrm{~cm}$ step. The results of this experiment are shown in Table 2 and

Figure 18.

Table 2. Effect of distance changes on the size of projected object.

\begin{tabular}{|c|c|c|c|c|c|}
\hline \multirow{2}{*}{$\begin{array}{c}\text { Original } \\
\text { object size } \\
(\mathbf{c m})\end{array}$} & $\mathbf{4 0} \mathbf{~ c m}$ & $\mathbf{3 5} \mathbf{~ c m}$ & $\mathbf{3 0} \mathbf{~ c m}$ & $\mathbf{2 5} \mathbf{~ c m}$ & $\mathbf{2 0} \mathbf{~ c m}$ \\
\cline { 2 - 6 } & 2.5 & 2.5 & 2.5 & 2.5 & 2.5 \\
\hline 5 & 2.25 & 2.25 & 2.25 & 2.25 & 2.25 \\
\hline 4.5 & 2 & 2 & 2 & 2 & 2 \\
\hline 4 & 1.75 & 1.75 & 1.75 & 1.75 & 1.75 \\
\hline 3.5 & 1.5 & 1.5 & 1.5 & 1.5 & 1.5 \\
\hline 3 & & & & & \\
\hline
\end{tabular}

The length of the projected object is smaller than the original object based on the results in Table 2. The magnification needs to implemented in order to equalize the object size between the original and projected. The magnification value can be obtained from the ratio of the original object length $h_{o}$ with the projected object length $h_{p}$ which is shown by Eqution 7. The correlation between $h_{o}, h_{p}$, and distance $(d)$ refers to

Figure 11. 


$$
M=\frac{h_{o}}{h_{p}}
$$

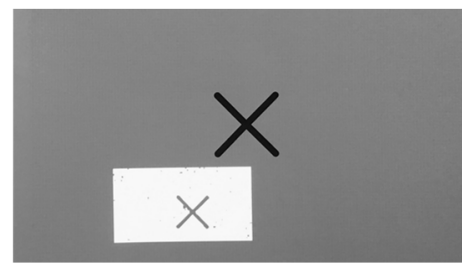

(a)

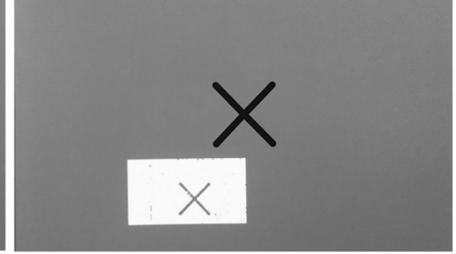

(b)

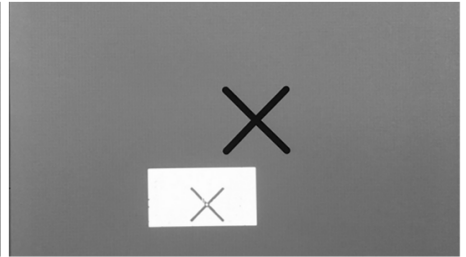

(c)

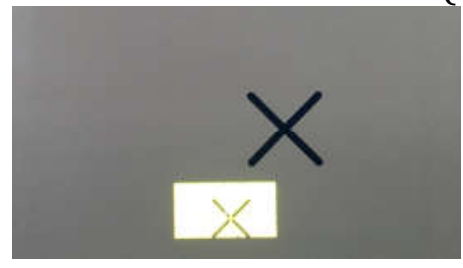

(d)

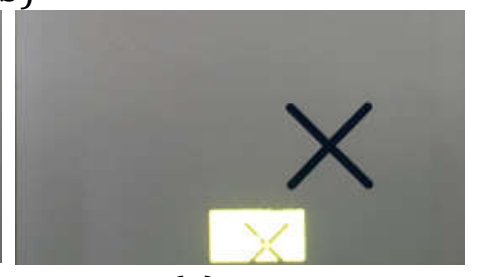

(e)

Figure 18. The projection results of $5 \mathrm{~cm}$ object length. The projection distance is $40,35,30,25$ and $20 \mathrm{~cm}$ shown in (a), (b), (c), (d), and (e) respectively.

In this case, the magnification value $M$ obtained from Equation 7 is $2 \mathrm{x}$ and after the old projected image was magnified, the length of projected object now has the same length as the original. The magnification process was performed before setting the region of interest (ROI) thus the frame size does not change as shown in Figure 19.

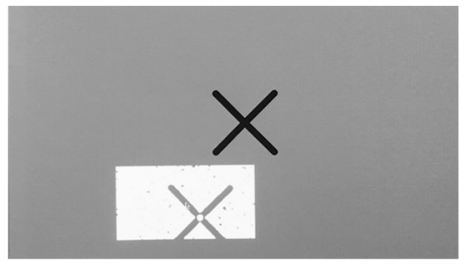

(a)

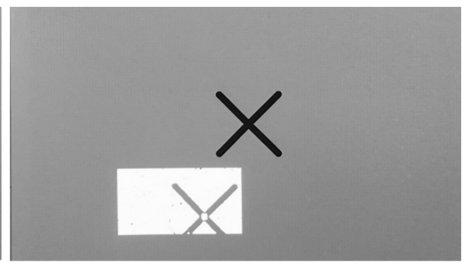

(b)

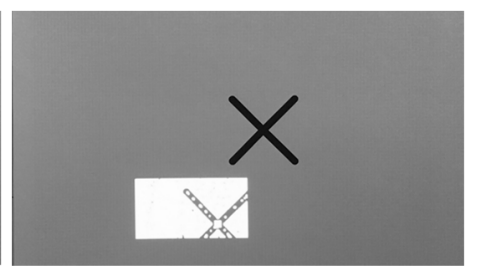

(c)

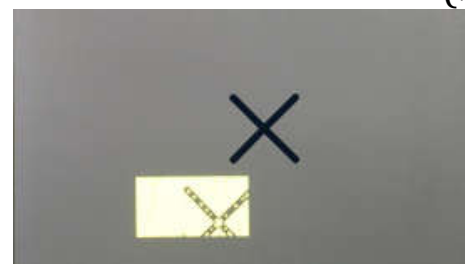

(c)

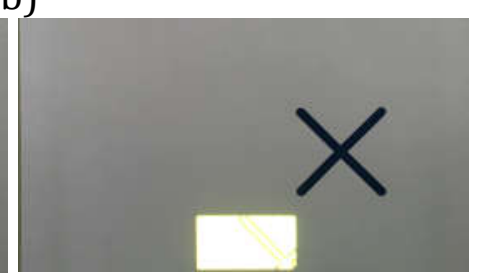

(d)

Figure 19. Projection results from two times magnification from the previous image. The projection distance is 40, 35, 30, 25 and $20 \mathrm{~cm}$ shown in (a), (b), (c), (d), and (e) respectively.

The same size between the original object and the projection object has been obtained, then further observation is needed to find out the characteristics of the projected location if there is a change in distance. 
Figure 20 shows the location of the processed frame in the projector view. In this experiment, $\mathrm{X}$ and $\mathrm{Y}$ locations of processed frame were tuned manually until there is no shift in projection at a distance of $20-40 \mathrm{~cm}$ with a 5 $\mathrm{cm}$ step. The results are shown in Table 3 where distance significantly affects the $\mathrm{X}$ and $\mathrm{Y}$ location. The characteristics for changes in distance $(d)$ to $\mathrm{X}$ and Y location have the polynomial curve as shown in

Figure 21. A regression by using data in Table 3 was performed in order to achieve adaptive projection. Based on these results, the value of $\mathrm{X}$ and $\mathrm{Y}$ based on the distance change could be approximated by the second order polynomial which is shown in Equation 8 and Equation 9.

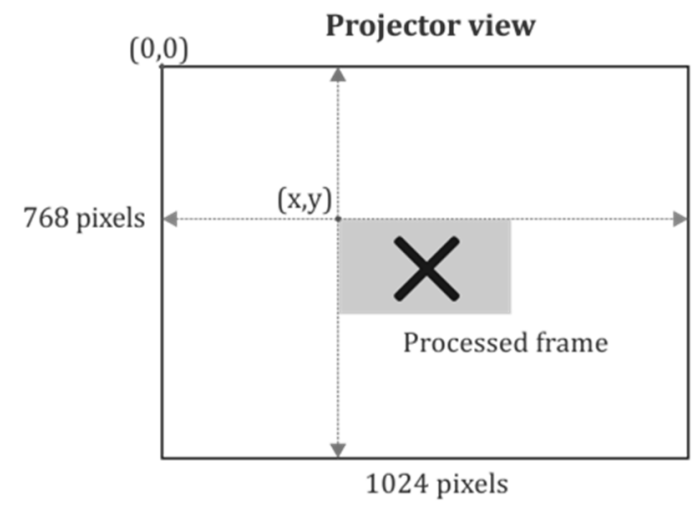

Figure 20. Processed frame in projector view illustration.

Table 3. The results of manually tuned $\mathrm{X}$ and $\mathrm{Y}$ location to find out the projection characteristics of the system.

\begin{tabular}{|c|c|c|}
\hline Distance (cm) & X location & Y location \\
\hline 40.3 & 448 & 40 \\
\hline 35.1 & 462 & 17 \\
\hline 30.1 & 480 & -13 \\
\hline 25.5 & 506 & -57 \\
\hline 20.5 & 540 & -120 \\
\hline
\end{tabular}




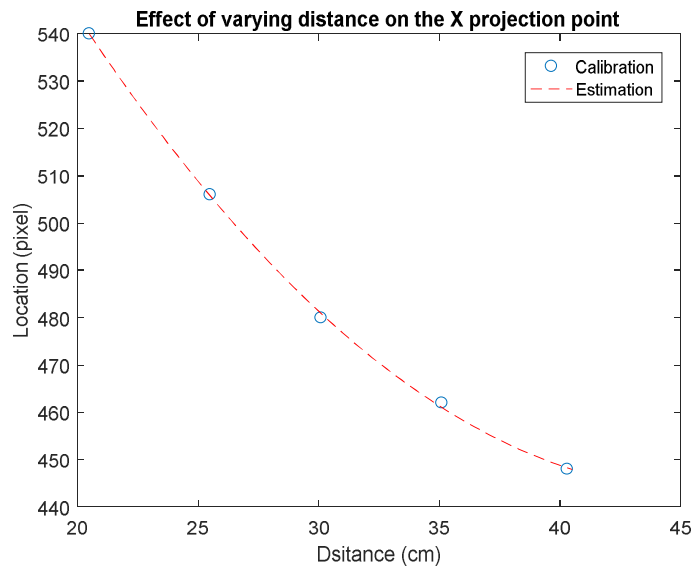

(a)

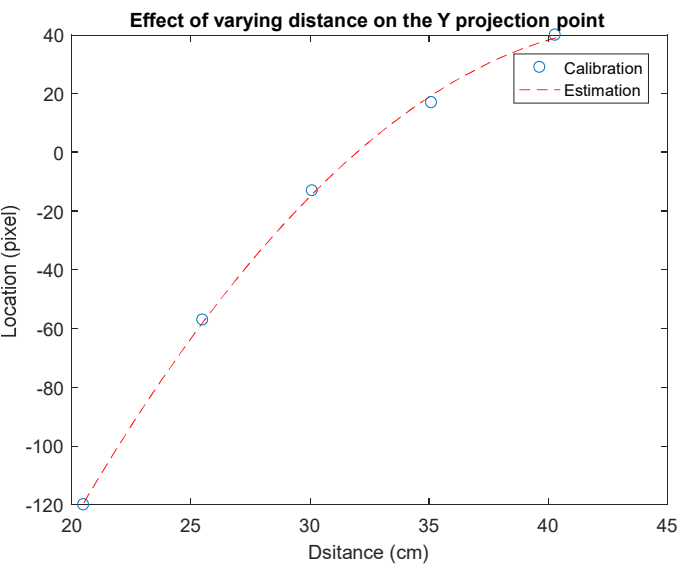

(b)

Figure 21. Visulaization of (a) $\mathrm{X}$ and (b) $\mathrm{Y}$ location in distance of $20-40 \mathrm{~cm}$

$$
\begin{gathered}
\mathrm{X}=0.1294 d^{2}-12.631 d+751.8 \\
\mathrm{Y}=-0.2775 d^{2}-25.072 d+494.78
\end{gathered}
$$

As shown in Figure 22, the projection location is able to adjust itself based on the distance between the object and device after Equation 8 and 9 applied. Re-measurement is performed after the adaptive projection process was applied. The comparison between the calibrated location and measured location is shown in Table 4.

Table 4. Comparison of locations $\mathrm{X}$ and $\mathrm{Y}$ between calibrations with the application of Equations 6 and 7

\begin{tabular}{|c|c|c|c|c|c|c|}
\hline \multirow{2}{*}{$\begin{array}{c}\text { Distance } \\
(\mathrm{cm})\end{array}$} & \multicolumn{2}{|c|}{$\begin{array}{c}\text { Calibration } \\
\text { location (pixel) }\end{array}$} & \multicolumn{2}{c|}{$\begin{array}{c}\text { Projection at } 0^{\circ} \\
\text { location (pixel) }\end{array}$} & \multicolumn{2}{c|}{ Difference (pixel) } \\
\cline { 2 - 7 } & $\mathrm{X}$ & $\mathrm{Y}$ & $\mathrm{X}$ & $\mathrm{Y}$ & $\mathrm{X}$ & $\mathrm{Y}$ \\
\hline 40.3 & 448 & 40 & 448 & 39 & 0 & 1 \\
\hline 35.1 & 462 & 17 & 461 & 19 & 1 & 2 \\
\hline 30.1 & 480 & -13 & 481 & -14 & 1 & 1 \\
\hline 25.5 & 506 & -57 & 506 & -58 & 0 & 1 \\
\hline 20.5 & 540 & -120 & 540 & -119 & 0 & 1 \\
\hline
\end{tabular}




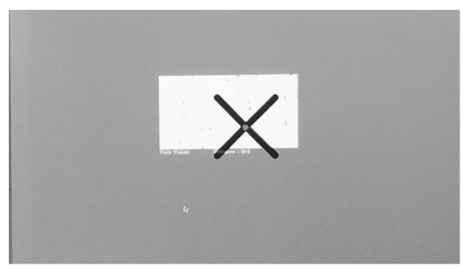

(a)

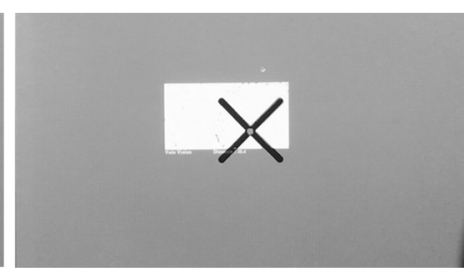

(b)

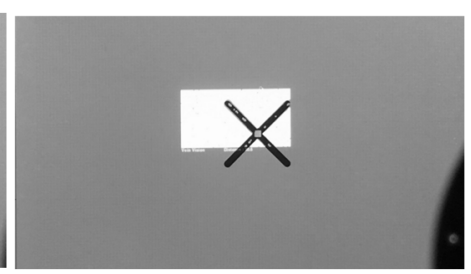

(c)

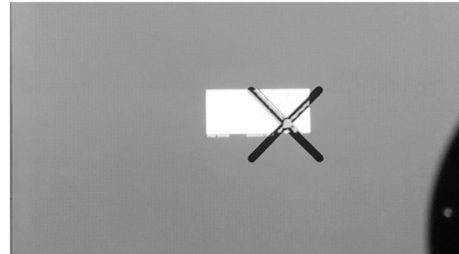

(d)

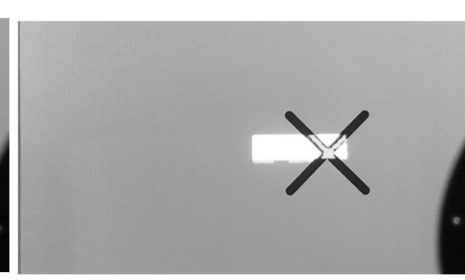

(e)

Figure 22. The back-projection results after applying Equation 8 and 9. The projection distance is $40,35,30,25$ and $20 \mathrm{~cm}$ shown in (a), (b), (c), (d), and (e) respectively.

The re-measurement after the application of adaptive projection has good results at X location. It has a difference of 0 pixels at a distance of 40.3, 25.5 , and $20.5 \mathrm{~cm}$, while at the other distance has a difference of 1 pixel. Whereas for $Y$ location has a pretty good result which has a difference of 1 pixel at all distances except at $35.1 \mathrm{~cm}$ has a difference of 2 pixels.

Apart from perpendicular angle $\left(0^{\circ}\right)$, some experiments also carried out at different angles. In this case, the specified angle limit is $\pm 5^{\circ}$. The results of back-projection at a distance of $20-40 \mathrm{~cm}$ with angular variations of $-10^{\circ},-5^{\circ}$, $0^{\circ},+5^{\circ}$, and $+10^{\circ}$ are shown in 
Table 5 .

EMITTER International Journal of Engineering Technology, ISSN: 2443-1168 
Table 5. Projection shift measurement results when the measurement angle turn from $-10^{\circ}$ to $10^{\circ}$

\begin{tabular}{|c|c|c|c|c|c|c|c|c|c|c|}
\hline \multirow{2}{*}{ Distance (cm) } & \multicolumn{10}{|c|}{ Rotation } \\
\cline { 2 - 12 } & \multicolumn{2}{|c|}{$-10^{\circ}$} & \multicolumn{2}{|c|}{$-5^{\circ}$} & \multicolumn{2}{|c|}{$0^{\circ}$} & \multicolumn{2}{|c|}{$5^{\circ}$} & \multicolumn{2}{|c|}{$10^{\circ}$} \\
\cline { 2 - 11 } & $\mathrm{X}$ & $\mathrm{Y}$ & $\mathrm{X}$ & $\mathrm{Y}$ & $\mathrm{X}$ & $\mathrm{Y}$ & $\mathrm{X}$ & $\mathrm{Y}$ & $\mathrm{X}$ & $\mathrm{Y}$ \\
\hline 40.3 & 0.4 & 0.8 & 0.1 & 0.4 & 0.1 & 0.3 & 0.2 & 0.4 & 0.4 & 0.8 \\
35.1 & 0.2 & 0.5 & 0.3 & 0.7 & 0.2 & 0.6 & 0.2 & 0.6 & 0.2 & 0.5 \\
30.1 & 0.1 & 0.1 & 0.2 & 0.3 & 0.2 & 0.2 & 0.2 & 0.3 & 0.1 & 0.1 \\
25.5 & 0.1 & 0.4 & 0.1 & 0.4 & 0.0 & 0.2 & 0.2 & 0.0 & 0.0 & 0.2 \\
20.5 & 0.0 & 0.1 & 0.0 & 0.1 & 0.0 & 0.1 & 0.0 & 0.1 & 0.1 & 0.3 \\
\hline
\end{tabular}

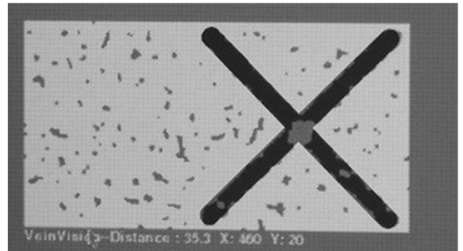

(a)

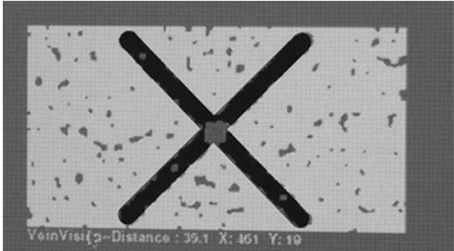

(b)

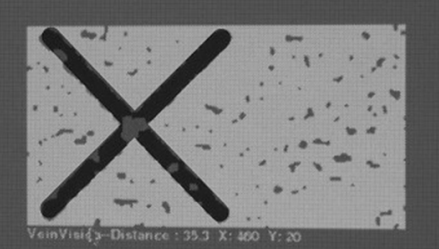

(c)

Figure 23. Projection result at $-5^{\circ}, 0^{\circ},+5^{\circ}$ that shown in (a), (b), (c) respectively in distance of $35.1 \mathrm{~cm}$

The application of adaptive projection produces good performance even though there are results with a considerable shift in all measurement angle at $35.1 \mathrm{~cm}$ as shown in Figure 23. In projections with angles of $-10^{\circ}$ and $10^{\circ}$, there was result that have the greatest shift value of $0.8 \mathrm{~mm}$ at $40.3 \mathrm{~cm}$ distance. A significant shift occurred at the farthest distance while at the closest distance there was no significant error. This phenomenon could be approached with the cosine ratio as follows:

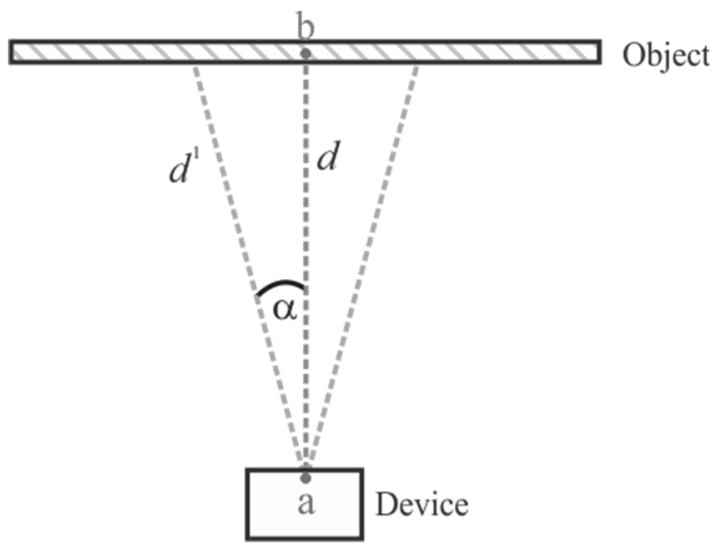

Figure 24. Cosine ratio to estimate distance when measuring in various angle In 
Figure 24, the distance between the device and the object $d^{1}$ can be obtained from Eq. (10) and (11), where: $d$ is the distance between the device (point a) and the object (point b).

$$
\begin{aligned}
& \cos \alpha=\frac{d}{d^{1}} \\
& d^{1}=\frac{d}{\cos \alpha}
\end{aligned}
$$

For example, the farthest distance in a measurement with a perpendicular angle is $40.3 \mathrm{~cm}$. By applying Equation 11, then the value of $d^{1}$ at $5^{\circ}$ is $40.5 \mathrm{~cm}$ while at $10^{\circ}$ is $40.9 \mathrm{~cm}$. From the closest distance of $20.5 \mathrm{~cm}$, it is also known that the value of $d^{1}$ at $5^{\circ}$ is $20.6 \mathrm{~cm}$ while at $10^{\circ}$ is $20.8 \mathrm{~cm}$. From these results, it is known that the rotation angle value $(\alpha)$ of the device directly proportional to the value of $d^{1}$. After the distance when turn at $5^{\circ}$ and $10^{\circ}$ were known, the projection location of $\mathrm{X}$ and $\mathrm{Y}$ can be found based

Figure 21. To prove this calculation, the following in Table 6-9 are the results of calculations and measurements for angles $+5^{\circ},-5^{\circ},+10^{\circ}$, and $-10^{\circ}$. Dist $\mathrm{C}$ represents the distance from the cosine ratio calculation while Dist $\mathrm{M}$ represents the measured distance. Xc and Yc are pixel locations obtained by Dist $\mathrm{C}$ according to the calibration values in

Figure 21, while $\mathrm{Xm}$ and $\mathrm{Ym}$ are measured pixel locations.

Table 6. Comparison of calibrated location with the measurement location at $-5^{\circ}$

\begin{tabular}{|c|c|c|c|c|c|c|c|c|c|c|}
\hline \multicolumn{7}{|c|}{ Calibration } & \multicolumn{7}{c|}{$5^{\circ}$} & \multicolumn{2}{c|}{ Difference } \\
\hline Dist $(\mathbf{c m})$ & $\mathbf{X}$ & $\mathbf{Y}$ & Dist C & Dist M & Xc & Yc & Xm & Ym & X & Y \\
\hline 40.3 & $\mathbf{4 4 8}$ & $\mathbf{4 0}$ & 40.5 & 40.4 & 447 & 41 & 448 & 39 & 1 & 2 \\
\hline 35.1 & $\mathbf{4 6 2}$ & $\mathbf{1 7}$ & 35.2 & 35.3 & 462 & 18 & 460 & 20 & 1 & 3 \\
\hline 30.1 & $\mathbf{4 8 0}$ & $\mathbf{- 1 3}$ & 30.2 & 30.2 & 479 & -12 & 480 & -13 & 1 & 1 \\
\hline 25.5 & $\mathbf{5 0 6}$ & $\mathbf{- 5 7}$ & 25.6 & 25.4 & 505 & -56 & 506 & -59 & 1 & 3 \\
\hline 20.5 & $\mathbf{5 4 0}$ & $\mathbf{- 1 2 0}$ & 20.6 & 20.6 & 539 & -119 & 539 & -118 & 0 & 1 \\
\hline
\end{tabular}

Table 7. Comparison of calibrated location with the measurement location at $+5^{\circ}$

\begin{tabular}{|c|c|c|c|c|c|c|c|c|c|c|}
\hline \multicolumn{7}{|c|}{ Calibration } & \multicolumn{7}{c|}{$+5^{\circ}$} & \multicolumn{2}{c|}{ Difference } \\
\hline Dist $(\mathrm{cm})$ & $\mathbf{X}$ & $\mathbf{Y}$ & Dist C & Dist M & Xc & Yc & Xm & Ym & X & Y \\
\hline 40.3 & $\mathbf{4 4 8}$ & $\mathbf{4 0}$ & 40.5 & 40.5 & 447 & 41 & 448 & 39 & 0 & 1 \\
\hline 35.1 & $\mathbf{4 6 2}$ & $\mathbf{1 7}$ & 35.2 & 35.3 & 462 & 18 & 460 & 20 & 1 & 3 \\
\hline 30.1 & $\mathbf{4 8 0}$ & $\mathbf{- 1 3}$ & 30.2 & 30.2 & 479 & -12 & 480 & -13 & 1 & 1 \\
\hline 25.5 & $\mathbf{5 0 6}$ & $-\mathbf{5 7}$ & 25.6 & 25.6 & 505 & -56 & 505 & -57 & 0 & 1 \\
\hline
\end{tabular}


20.5

\begin{tabular}{|l|l|l|}
$\mathbf{5 4 0}$ & $\mathbf{- 1 2 0}$ & 20.6 \\
\hline
\end{tabular}

20.7

539

$-119$

538

$-117$

1

2

Table 8. Comparison of calibrated location with the measurement location at $-10^{\circ}$

\begin{tabular}{|c|c|c|c|c|c|c|c|c|c|c|}
\hline \multicolumn{3}{|c|}{ Calibration } & \multicolumn{6}{|c|}{$-10^{\circ}$} & \multicolumn{2}{|c|}{ Difference } \\
\hline Dist (cm) & $\mathbf{X}$ & $\mathbf{Y}$ & Dist C & Dist M & $\mathrm{Xc}$ & Yc & $\mathrm{Xm}$ & $\mathrm{Ym}$ & $\mathrm{X}$ & Y \\
\hline 40.3 & 448 & 40 & 40.9 & 40.7 & 446 & 43 & 448 & 40 & 2 & 3 \\
\hline 35.1 & 462 & 17 & 35.6 & 35.5 & 461 & 19 & 460 & 22 & 1 & 2 \\
\hline 30.1 & 480 & -13 & 30.6 & 30.6 & 478 & -9 & 479 & -10 & 1 & 1 \\
\hline 25.5 & 506 & -57 & 25.9 & 25.8 & 503 & -53 & 504 & -55 & 0 & 2 \\
\hline 20.5 & 540 & -120 & 20.8 & 20.8 & 538 & -116 & 538 & -115 & 0 & 1 \\
\hline
\end{tabular}

Table 9. Comparison of calibrated location with the measurement location at $10^{\circ}$

\begin{tabular}{|c|c|c|c|c|c|c|c|c|c|c|}
\hline \multicolumn{9}{|c|}{ Calibration } & \multicolumn{1}{|c|}{$+10^{\circ}$} & \multicolumn{2}{c|}{ Difference } \\
\hline Dist $(\mathrm{cm})$ & $\mathbf{X}$ & $\mathbf{Y}$ & Dist C & Dist M & Xc & Yc & Xm & Ym & X & Y \\
\hline 40.3 & $\mathbf{4 4 8}$ & $\mathbf{4 0}$ & 40.9 & 40.9 & 446 & 43 & 447 & 40 & 1 & 3 \\
\hline 35.1 & $\mathbf{4 6 2}$ & $\mathbf{1 7}$ & 35.6 & 35.5 & 461 & 19 & 460 & 22 & 1 & 2 \\
\hline 30.1 & $\mathbf{4 8 0}$ & $\mathbf{- 1 3}$ & 30.6 & 30.6 & 478 & -9 & 479 & -10 & 1 & 1 \\
\hline 25.5 & $\mathbf{5 0 6}$ & $\mathbf{- 5 7}$ & 25.9 & 25.9 & 503 & -53 & 503 & -54 & 0 & 1 \\
\hline 20.5 & $\mathbf{5 4 0}$ & $\mathbf{- 1 2 0}$ & 20.8 & 20.9 & 538 & -116 & 537 & -114 & 1 & 2 \\
\hline
\end{tabular}

For example, there is a large difference in pixel location at a distance $d$ of $40.3 \mathrm{~cm}$ when measuring at $+10^{\circ}$. By applying Equation 11, the value of calculated $d^{1}$ at $10^{\circ}$ is $40.9 \mathrm{~cm}$. Equation 8 and Equation 9 are applied to measured $d^{1}$ to get the X location which is at 446.57 pixel and the Y location at 40.18 pixel. However, since 1 pixel is the smallest unit, the result will be rounded to 447 and 40 then it has a difference of 1 pixel of $X$ and 3 pixels of $Y$ from the calibration results.

In measurement at $-5^{\circ}$, there is also a considerable error at a distance of $25.5 \mathrm{~cm}$. Regarding from the results of Equation 11, the value of calculated $d^{1}$ at $-5^{\circ}$ is $25.597 \mathrm{~cm}$. In the same way Equation 8 and Equation 9 are applied to measured $d^{1}$, then the X location is obtained at 506.347 pixel and the $\mathrm{Y}$ location at -58.903 pixel. After through the rounding process, $\mathrm{X}$ location becomes 506 and Y location becomes -59 . The value has a difference of 1 pixel with $\mathrm{X}$ and 3 pixels with $\mathrm{Y}$ from the results of calibration. Based on the results from

Figure 17, 340 pixels frame length has a different projection size in $\mathrm{cm}$ when projected at multi-distance $(20-40 \mathrm{~cm})$. The projected frame length against distance can be approximated by Equation 12 where $h_{p}$ is estimation of the projected frame length and $d$ is the distance between device and object.

$$
h_{p}=0.22 \times d+0.5
$$


From the previous discussion, the projection at $+10^{\circ}$ with a distance of 40.3 $\mathrm{cm}$ have a difference of $0.8 \mathrm{~mm}$ at Y location. When the Equation 12 is applied, projection frame length at $40.9 \mathrm{~cm}$ is $9.5 \mathrm{~cm}$ thus 1-pixel size equal to the projected frame length $(9.5 \mathrm{~cm})$ divided by frame length (340 pixel). Based on the results of calculations and measurements from

Table 8, the difference between measured Y with calibrated $Y$ is 3 pixels. If 1 pixel at that distance represents $0.23 \mathrm{~mm}$, then the calculation of projection shift is $0.72 \mathrm{~mm}$. The measurement results are very close to the calculation which only has a difference of $0.08 \mathrm{~mm}$.

The projection at $+5^{\circ}$ with a distance of $25.5 \mathrm{~cm}$ also produces a difference of 3 pixels at $Y$ location. After equation 12 is applied, the projected frame length at that distance is $6.11 \mathrm{~cm}$ and 1 pixel represents $0.18 \mathrm{~mm}$. If there is a difference of 3 pixels, the calculation of the projected shift is $0.54 \mathrm{~mm}$ while the measurement result is $0.4 \mathrm{~mm}$. From these two samples, it can be seen that the difference of 1 pixel over a long distance will produce a greater shift than the difference of 1 pixel at close range. The difference that exceeds 1 pixel in Table 6-9 occurs due to the reading of the ultrasonic sensor stated as the measured distance has an error from the calculation of distance using the cosine ratio.

After this system was developed and several tests were carried out, a comparison of previous studies was conducted to see the differences in each study from several aspects. As shown in Table 10, there are 5 aspects that can be compared: construction, projection distance, computation, portable system, and projection shift. It can be seen that the proposed method in this paper has advantages in easy construction, able to perform multi-distance projection, low power computation system using a single board computer and portable system. From the projection shift aspect, a good result was provided by Zeman within $<0.1 \mathrm{~mm}$ projection shift. In this proposed method, the projection shift ranging from 0-0.8 $\mathrm{mm}$ because the projection distance also ranges from 20$40 \mathrm{~cm}$. Since this proposed method able to perform multi-distance projection, the projection shift is affected by the distance reading. Furthermore, the distance reading must be optimized to reduce the projection shift.

Table 10. Comparison between the proposed method with related works

\begin{tabular}{|c|c|c|c|c|}
\hline Construction & $\begin{array}{c}\text { Zeman [3] } \\
\text { mechanical } \\
\text { construction }\end{array}$ & Dai [4] & Ai [5] & $\begin{array}{c}\text { Proposed } \\
\text { Method }\end{array}$ \\
\hline $\begin{array}{c}\text { Projection } \\
\text { distance }\end{array}$ & $\begin{array}{c}\text { Fixed at 50.8 } \\
\mathrm{cm}\end{array}$ & $\begin{array}{c}\text { Fixed (not } \\
\text { mentioned) }\end{array}$ & Fixed at 35 cm & $\begin{array}{c}\text { Multi-distance } \\
(20-40 \mathrm{~cm})\end{array}$ \\
\hline Computation & PC & PC & PC & $\begin{array}{c}\text { Single Board } \\
\text { Computer }\end{array}$ \\
\hline $\begin{array}{c}\text { Portable } \\
\text { system }\end{array}$ & No & No & No & yes \\
\hline $\begin{array}{c}\text { Projection } \\
\text { shift }\end{array}$ & $<0.1 \mathrm{~mm}$ & $0.8 \mathrm{~mm}$ & $\begin{array}{c}\text { No } \\
\text { measurement }\end{array}$ & $0-0.8 \mathrm{~mm}$ \\
\hline
\end{tabular}




\begin{tabular}{|l|l|l|c|l|}
\hline & & & $\begin{array}{c}\text { on projection } \\
\text { shift }\end{array}$ & \\
\hline
\end{tabular}

\section{CONCLUSION}

We have presented a multi-distance veins visualization system based on a single axis camera-projector system. In this research, the veins visualization system consists of acquisition, preprocessing, segmentation and backprojection stage to visualize the veins image on the skin. A veins image that captured using reflection NIR does not provide a clear veins pattern and it needs to enhance in preprocessing stage. Using the combination of adaptive thresholding and morphology operation, the veins pattern can be obtained in the segmentation stage and ready to be projected on the skin. Scale, distance, and location of the processed veins image were tuned in the back-projection stage to obtain the correct projection result in multi-distance. By combining several image processing algorithms, this system able to process 1 frame with 0.065 second or equal to 15 frames per second. The proposed method to achieve multi-distance back-projection was successfully carried out in distance of $20-40 \mathrm{~cm}$ with maximum projection shift of $0.6 \mathrm{~mm}$ in perpendicular position. While in various angle measurements, the projection shift at $\pm 5^{\circ}$ still can be tolerated because the maximum is $0.72 \mathrm{~mm}$. Where in $\pm 10^{\circ}$ measurement, the maximum projection shift increases up to $0.8 \mathrm{~mm}$. Furthermore, because this projection method is distance-based, it is necessary to develop a method to improve the accuracy of distance reading.

\section{REFERENCES}

[1] Min Joung Kim, Joon Min Park, Nuga Rhee, Sang Mo Je, Seong Hee Hong, Young Mock Lee, Sung Phil Chung and Seung Ho Kim, Efficacy of VeinViewer in pediatric peripheral intravenous access: A randomized controlled trial, Eur. J. Pediatr., vol. 171, no. 7, pp. 11211125, 2012.

[2] Simon Juric and Borut Zalik, An innovative approach to near-infrared spectroscopy using a standard mobile device and its clinical application in the real-time visualization of peripheral veins, BMC Med. Inform. Decis. Mak., vol. 14, no. 1, pp. 1-9, 2014.

[3] Herbert D. Zeman, Prototype vein contrast enhancer, Opt. Eng., vol. 44, no. 8, p. 086401, 2005.

[4] Xaobin Dai, Ya Zhou, Xiaoming Hu, Meiqing Liu, Xia Zhu, and Zhaogou $\mathrm{Wu}$, A fast vein display device based on the camera-projector system, IST 2013 - 2013 IEEE Int. Conf. Imaging Syst. Tech. Proc., pp. 146-149, 2013.

[5] Danni Ai, Jian Yang, Jingfan Fan, Yitian Zhao, Xianzheng Song, Jianbing Shen, Ling Shao and Yongtian Wang, Augmented reality based realtime subcutaneous vein imaging system, Biomed. Opt. Express, vol. 7, no. 7, p. 2565, 2016.

[6] I Putu Adi Surya Gunawan, Riyanto Sigit, and Agus Indra Gunawan, Vein 
Visualization System Using Camera and Projector Based on Distance Sensor, in 2018 International Electronics Symposium on Engineering Technology and Applications (IES-ETA), 2018, pp. 150156.

[7] Scott Prahl, Optical Arbsorption of Hemoglobin, 1999. [Online]. Available: https://omlc.org/spectra/hemoglobin/. [Accessed: 21-Jan2018].

[8] Donghoon Kim, Yujin Kim, Siyeop Yoon, and Deukhee Lee, Preliminary study for designing a novel vein-visualizing device, Sensors (Switzerland), vol. 17, no. 2, 2017.

[9] Liukui Chen, Jing Wang, Shiyu Yang, and Haibo He, A Finger Vein Image-Based Personal Identification System with Self-Adaptive Illuminance Control, IEEE Trans. Instrum. Meas., vol. 66, no. 2, pp. 294304, 2017.

[10] Yiding Wang, Yun Fan, Weiping Liao, Kafeng Li, Lik-Kwan Shark, and Martin R. Varley, Hand vein recognition based on multiple keypoints sets, Proc. - 2012 5th IAPR Int. Conf. Biometrics, ICB 2012, pp. 367-371, 2012.

[11] Anagha. B. Bawase and P. S. D. Apte, Infrared Hand Vein Detection System, pp. 48-52, 2015.

[12] Adam Shidqul Aziz, Riyanto Sigit, Achmad Basuki, and Taufik Hidayat, Cardiac Motions Classification on Sequential PSAX Echocardiogram, Indones. J. Electr. Eng. Comput. Sci., vol. 12, no. 3, pp. 1289-1296, 2018.

[13] Galih Hendra Wibowo, Riyanto Sigit, and Ali Ridho. Barakbah, Javanese Character Feature Extraction Based on Shape Energy, EMITTER International Journal Engineering Technology, vol. 5, no. 1, pp. 154-170, 2017

[14] Muqing Zhou, Zhaogou Wu, Difan Chen, and Ya Zhou, An improved vein image segmentation algorithm based on SLIC and Niblack threshold method, Proc. SPIE 9045, 2013 Int. Conf. Opt. Instruments Technol. Optoelectron. Imaging Process. Technol., vol. 9045, p. 90450D, 2013.

[15] George J. Tserevelakis, Ilianna Vrouvaki, Panagiotis Siozoz, Krystallia Mellesanaki, Kostas Hatzigiannakis, Costas Fotakis, and Giannis Zacharakis, Photoacoustic imaging reveals hidden underdrawings in paintings, Sci. Rep., vol. 7, no. 1, pp. 1-11, 2017.

[16] Rebecca Sharp, Melita Cummings, Jessie Childs, Andrea Fielder, Antonia Mikocka-Walus, Carol Grech and Adrian Esterman, Measurement of Vein Diameter for Peripherally Inserted Central Catheter (PICC) Insertion: An Observational Study, J. Infus. Nurs., vol. 38, no. 5, pp. 351-357, 2015. 